\title{
Training Culturally Congruent Health Coaches to be Socially- Embedded Support on Physical Activity among Older Asian Americans: Phase One Report
}

\author{
Meriam Caboral-Stevens ${ }^{1}$, and Tsu-Yin $\mathrm{Wu}^{2}$ \\ ${ }^{1}$ Center for Health Disparities Innovations and Studies, Eastern Michigan University \\ Ypsilanti, MI USA \\ Email: msteve37 [AT] emich.edu \\ ${ }^{2}$ Center for Health Disparities Innovations and Studies, Eastern Michigan University \\ Ypsilanti, MI USA \\ Email: twu [AT] emich.edu
}

\begin{abstract}
There is a reported heterogeneity in burden with cardiovascular disease (CVD) among Asian Americans subgroups. Physical inactivity is an identified key risk factor for CVD, across age and race/ethnic backgrounds. Latest data showed that $42 \%$ of Asian Americans are physically inactive. Health coaching is a rapidly emerging primary or supplemental intervention to address issues related to preventable chronic illness, including physical inactivity. Hence, the purpose of this paper is to describe Phase One of this project - training of culturally congruent Health Coaches as socially embedded social support to promote physical activity to their older Asian communities. A 3-day training curriculum was developed based on the four pillars of Health Coaching and the Transtheoretical Model of Change. Fifteen Asian Americans Health Coaches were trained using this curriculum. Fifteen health coaches completed the 3-day training. Phase Two of this project is ongoing and results may provide information on the long-term effects of health coaching among older Asian Americans.
\end{abstract}

Keywords - health coaches, socially-embedded social support, physical activity, older Asian Americans

\section{INTRODUCTION}

Cardiovascular disease (CVD) is the second leading cause of death among Asian Americans [1]. Physical inactivity is an identified risk factor, across age and race/ethnic backgrounds, in developing chronic diseases including CVD [2]. It is reported that $42 \%$ of Asian Americans are physically inactive compared to non-Hispanic whites (39\%) [3]. Given the heterogeneity in CVD burden among Asian American subgroups, it is important to develop strategies that are rooted in cultural values [4], and addresses the challenges related to cultural differences including language barriers.

The Center for Health Disparities at Eastern Michigan University received funding to reduce disparities among older Asian Americans in the state of Michigan by training culturally congruent Health Coaches (HC) as socially embedded support to promote physical activity. This project is funded for two years and has two phases - to train culturally congruent health coaches and for HCs to recruit community members to follow up for one year. The purpose of this paper is to describe phase one of the project - training of culturally congruent HCs. Culturally congruent Health Coach is defined as individuals self-identified to be a member of Asian American subgroup who speaks, writes, and understands the native language and cultures, has close ties within the community, and is willing to provide long-term support to the members of their community.

\section{THEORETICAL MODEL}

The Four Pillars of Health Coaching [5] was the foundational model used in coaching instruction and practices. The four pillars are mindful presence, authentic communication, self-awareness, and a safe and sacred place. Mindful presence is practicing mindfulness in relation to another person. Authentic communication builds upon motivational interviewing, appreciative inquiry, and non-violent communications. The third pillar is self-awareness, in which HCs are to become aware of feelings and reactions that may arise internally during sessions with the members. Lastly, safe and sacred space refers to the creation of a safe milieu when meeting with the community member [5]. In addition, the training included contents to increase the HCs' baseline knowledge of CVD and behavior change. The Transtheoretical Model (TTM) of Change by Proschaska and DiClementi [6], was used to develop the content of the training curriculum 
on behavior change (Table 1). HCs were taught that the first step in promoting healthy behavior is understanding and applying the five stages of behavior change - precontemplation, contemplation, preparation, action, and maintenance [6].

Table 1. Curriculum Contents based on Transtheoretical Model of Change

\begin{tabular}{|c|c|c|c|c|}
\hline TTM & $\begin{array}{l}\text { Precontemplation/ } \\
\text { Contemplation } \\
\text { [Awareness] }\end{array}$ & $\begin{array}{l}\text { Contemplation/ } \\
\text { Preparation } \\
\text { [Knowledge] }\end{array}$ & $\begin{array}{l}\text { Action } \\
\text { [Skills] }\end{array}$ & Maintenance \\
\hline Contents & $\begin{array}{l}\text { Risk factors } \\
\text { Assessment Tools } \\
\text { Mindfulness } \\
\text { Self-reflection } \\
\text { Ethical Consideration }\end{array}$ & $\begin{array}{l}\text { Introduction to CVD } \\
\text { Physical Activity } \\
\text { Guidelines for older } \\
\text { adults } \\
\text { Difference between PA } \\
\text { and Exercise } \\
\text { Culturally-tailored } \\
\text { Strategies to Improve CV } \\
\text { Health }\end{array}$ & $\begin{array}{l}\text { ExerStart } \\
\text { Return demonstration } \\
\text { on obtaining } \\
\text { anthropometric } \\
\text { measurements }\end{array}$ & $\begin{array}{l}\text { Communication } \\
\text { messaging and } \\
\text { follow-up }\end{array}$ \\
\hline
\end{tabular}

TTM- transtheoretical model of change; CVD- cardiovascular disease; PA- physical activity

\section{ETHICAL CONSIDERATION}

The entire project received approval from our university's Human Subjects Review Committee [IRB Study \#: UHSRC-FY20-21-220]. Informed consent was not required for Phase One - training of Health Coaches since participation is voluntary. However, Phase Two of the project requires for Health Coaches to obtain informed consent from community members.

\section{TRAINING CURRICULUM DEVLEOPMENT}

\section{Review of the Literature}

Health coaching is a rapidly growing primary and supplemental strategy to address issues related to preventable chronic illness [7]. It is a patient-centered approach, in which patients are to set their own goals, encourage selfdiscovery, and develop accountability of their health behavior [7]. There is evidence that showed short-term health coaching is effective in behavior change among diabetic patients [8], and weight reduction among overweight and obese children using a mobile App [9]. Health and wellness coaches are typically health care professionals trained in behavioral change theory, motivational strategies, and communication techniques in order to help individuals develop intrinsic motivations to create a sustainable change in the person's behaviors in order to promote health and well-being [8]. However, challenges with relying on health care professionals as HCs for Asian Americans include lack of time, communication and language barrier, may not have community ties, and lack of trust among minority groups. Therefore, providing basic training for culturally congruent HCs may be effective in initiating and sustaining behavior change.

\section{Curriculum}

We developed a 3-day Health Coach training curriculum specific only for the purpose of this project. The first two days of training were delivered virtually via Zoom because of the ongoing COVID-19 pandemic. Each session was 90 minutes long. The third day of training was delivered face-to-face at a community center in order to provide the HCs with the materials needed to recruit and enroll community members as well as to practice skills in obtaining anthropometric measures, such as blood pressure, body mass index, and body fat. Ethical consideration was also included in the third day of training. Table 2 presents the content of each day's training. Specifically, the content of the training was focused on Asian American cultures, including disease burden, risk factors, screening tools, and culturally-tailored health promotions. 
Table 2. 3-Day Training Curriculum Contents

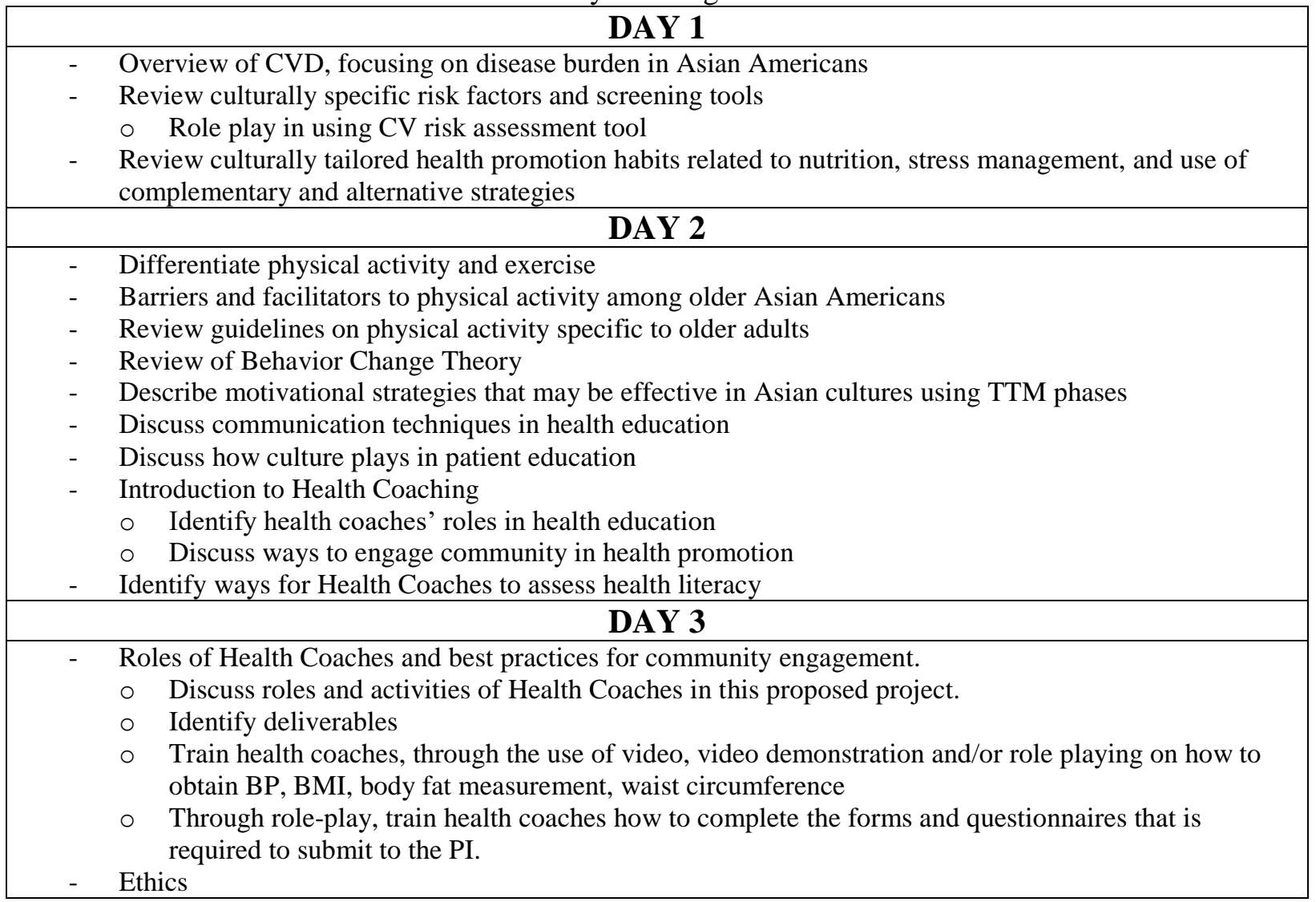

\section{Health Coaches}

HCs were recruited with the help of the Center's Asian Communities toward Innovative Visionary Environment (ACTIVE) Coalition, composed of leaders and stakeholders from various Asian communities. Each Asian community representative identified individuals who were a good fit as HC.

Twenty-five HCs were identified, and 15 HC participated in the 3-day training. Conflict with work and school schedules were the reasons for not participating. Trained HCs are composed of four Chinese, four Filipinos, two Bangladeshi, one each from Burmese, Indian, Nepalese, Thai, and Vietnamese communities. Pre-and-post-tests were administered at each training session. Overall HCs found the training as good (22\%) and excellent (78\%) using a scale of 1-5 (poor-excellent). Over 50\% of the HCs noted the method of presentation as excellent. Majority of the HCs reported that the training was organized, delivered expected information, duration of the training was sufficient, contents were relevant to the outcomes of the program and presented the contents effectively.

\section{DISCUSSION}

Living a healthy lifestyle has shown to reduce or delay the development of chronic illness and improve a person's quality of life (CDC, 2021). However, engaging and sustaining healthy behavior among minority groups is complex and may require support from within their community. Trained HC as socially embedded support may bridge the gap in health disparities in physical activity among older Asian Americans.

In summary, we presented Phase One of our project. Phase Two is ongoing and results of phase two may provide information on the long-term effects of culturally congruent HCs on physical activity among older Asian Americans.

\section{ACKNOWLEDGEMENT}

This work was supported by the Michigan Health Endowment Fund Community Health Impact [Grant number $R-2101-147818$ ].

\section{REFERENCES}

[1] Heron M. "Deaths: leading causes for 2016.” National Vital Statistics Report. Vol. 67, no. 6, pp. 1-77, 2018. https://www.cdc.gov/nchs/data/nvsr/nvsr67/nvsr67_06.pdf 
[2] Center for Disease Control and Prevention. "Lack of Physical activity". 2020 https://www.cdc.gov/chronicdisease/resources/publications/factsheets/physical-activity.htm

[3] U. S. Department of Health and Human Services Office of Minority Health. "Obesity and Asian Americans" 2020. https://minorityhealth.hhs.gov/omh/browse.aspx?lvl=4\&lvlid=55

[4] Domingo, JLB., Gavero, G, \& Braun, KL. "Strategies to increase Filipino American participation in CV health promotion: A systematic review. Preventing Chronic Disease vol. 15, pp. 170294, 2018. https://doi.org/10.5888/pcd15.170294

[5] Lawson K. "The four pillars of health coaching: Preserving the heart of a movement." Global Advances in Health and Medicine, vol. 2 no. 3, pp. 6-8, 2013. https://doi.org/10.7453/gahmj.2013.038

[6] Prochaska JO \& DiClemente CC. "Stages and processes of self-change of smoking: Toward an integrative model of change." Journal of Consulting and Clinical Psychology, vol. 51, no. 3, pp. 390-395 1983. http://ezproxy.emich.edu/login?url=https://www.proquest.com/scholarly-journals/stages-processes-self-changesmoking-toward/docview/614330641/se-2?accountid=10650

[7] Ching-Ling L, Li-Chi H, Yao-Tsung C, Ruey-Yu C, Yang S. "Effectiveness of health coaching in diabetes control and lifestyle improvement: A randomized-controlled trial." Nutrients, vol. 13, no. 11, pp. 3878, 2021. http://ezproxy.emich.edu/login?url= https:// www. proquest.com/scholarly-journals/effectiveness-health-coachingdiabetescontrol/docview/ 2602147216/se-2

[8] Wolever RQ, Simmons LA, Sforzo GA, et al. "A Systematic Review of the Literature on Health and Wellness Coaching: Defining a Key Behavioral intervention in Healthcare." Global Advances in Health and Medicine, vol. 2, no. 4, pp. 38-57, 2013. https://doi.org/10.7453/gahmj.2013.042

[9] Cueto V, Wang CJ, \& Lee MS. "Impact of a mobile App-Based health coaching and behavior change program on participant engagement and weight status of overweight and obese children: Retrospective cohort study." JMIR mHealth and uHealth, vol. 7, no. 11), 2019.

http://ezproxy.emich.edu/login?url=https://www.proquest.com/scholarly-journals/impact-mobile-app-based-healthcoaching-behavior/docview/2511255960/se-2 